\title{
Dr. Jorge Espino Vela (1921-2019)
}

\section{Dr. Jorge Espino Vela, M.D. (1921-2019)}

Francisco J. Espinosa-Rosales, Cinthya Tapia-Ponce, Edgar Amín Rivas-Zúñiga

Sin duda hay personas que marcan a las instituciones, pero sobre todo marcan a los que tienen la fortuna de conocerlas, no sólo por sus contribuciones y enseñanzas en el ámbito laboral, científico o académico, sino también por su gran calidad humana.
Espino... "lo que no está escrito no vale" ... así que por eso, en memoria del maestro, decidimos publicar un texto que nos dejó en el que relata la fundación y la labor de nuestra revista, enfatizando la importancia de publicar y difundir el conocimiento por medio de la escritura.
Para todos los que trabajamos en la revista Acta Pediátrica de México, y para muchos otros trabajadores del Instituto Nacional de Pediatría que tuvimos la fortuna de conocer al Dr. Jorge Espino Vela, sabemos que fue un ejemplo de rectitud, honradez y liderazgo, fue maestro de muchos pediatras y cardiólogos que por aquí han pasado y que mantendrán vivo su legado por muchos años, ya que marcó una época en la cardiología y en la edición.

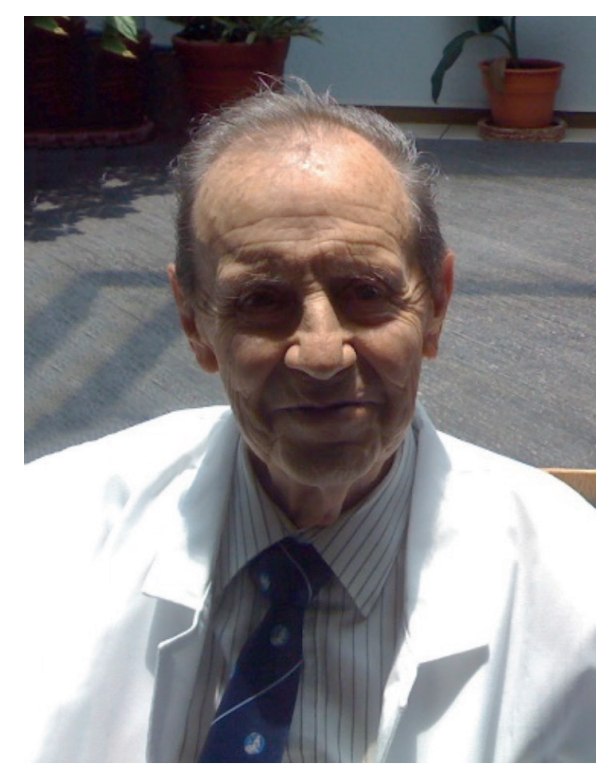

\section{Con tristeza comunicamos}

que el Dr. Jorge Espino Vela falleció el pasado 24 de marzo de 2019, pero al mismo tiempo celebramos su vida en estas líneas a manera de homenaje por su siempre desinteresada labor médica, educativa y científica; porque aunque estamos seguros de que su trabajo seguirá trascendiendo a través de sus alumnos, pacientes y compañeros, como lo decía el Dr.
Su entrañable amigo, el Dr. Samuel Zaltzman, nos confesó que al Dr. Espino no le gustaban los textos biográficos como homenaje. En este texto, queda plasmada la visión de un médico y escritor excepcional que dejó un amplio acervo en el saber de la cardiología pediátrica, pero que además fundó y vio crecer la revista Acta Pediátrica de México. Ahora, desde donde esté, seguramente seguirá viendo los frutos de su trabajo y esfuerzo, porque esta revista continúa siendo un órgano de difusión científica primordial para todos los pediatras de México y Latinoamérica, en beneficio de la salud infantil.

$* * *$

Era director del Instituto Nacional de Pediatría el Dr. Francisco Beltrán Brown, mi compañero en la escuela preparatoria y más tarde en la escuela de medicina. Desde entonces hemos 
conservado una estrecha, fraternal amistad. Tenemos la misma edad: 6 juventudes de 15 años cada una. Esto me lleva a recordar las 4 edades del hombre: la infancia, la adolescencia, la edad adulta y iqué bien te ves!

Vine al Instituto a saludar al Dr. Beltrán en 1980. Todavía no me había incorporado al INP (lo haría un año después al Servicio de Cardiología). En esa visita, el Dr. Beltrán me dijo que había pensado que el INP debería tener una revista, un órgano oficial. Me pidió que de hacerse realidad esa idea, fuera yo el encargado de fundarla y dirigirla como editor. Acepté la idea y el encargo.

Ese año, se publicó el primer número de la revista a la que le dimos el nombre de: "Acta Pediátrica de México". En esa época el INP se Ilamaba

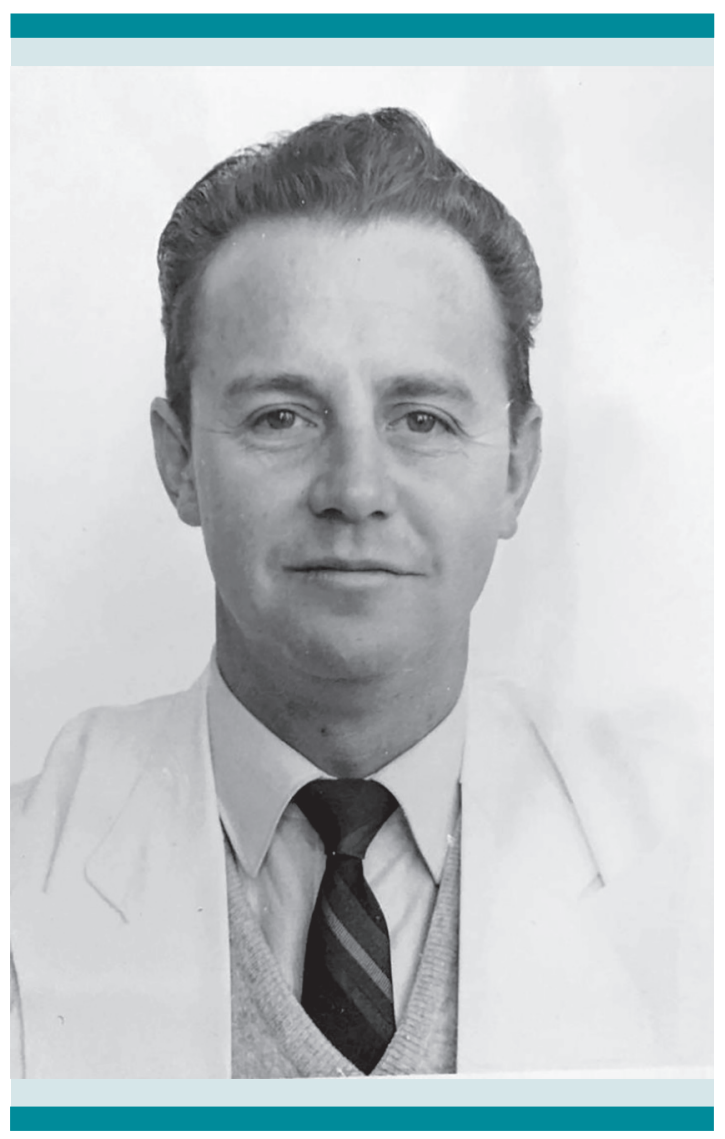

Sistema Nacional para el Desarrollo Integral de la Familia (DIF). Desde 1980 y hasta 1991 se publicaban cuatro fascículos al año.

El Dr. Beltrán se retiró hace algunos años, pero antes de terminar su gestión como director (1977-1980), tuvo una entrevista con el entonces presidente López Portillo, a quién le comentó que el DIF, por su cualidad como hospital, por su excelente personal médico y por sus recursos, tenía las condiciones idóneas para ser un instituto. Eso sucedería años después. Al dejar su puesto de director, el Dr. Beltrán fue nombrado Subsecretario de Salud, cuando era secretario el Dr. Mario Calles.

Nuestra revista recibía cada vez más artículos, lo que reflejaba el creciente interés de nuestros pediatras por esta publicación y su deseo de comunicar su experiencia al universo de pediatras de México, por eso fue necesario, a partir de 1991, publicar seis fascículos anualmente.

Durante varios años Acta Pediátrica de México fue financiada por el INP. Hubo dos o tres compañías editoriales encargadas sucesivamente de publicar y distribuir la revista. Diversos problemas obligaron a cambiar de compañía editorial. Desde el año 2001 hemos tenido la fortuna de contar con los servicios inmejorables de la empresa "Edición y Farmacia". Esta empresa es dirigida por el Dr. Enrique Nieto, gracias a él y a su equipo, Acta Pediátrica de México ha aparecido puntualmente en forma ininterrumpida con una excelente presentación. Por lo demás, la calidad de los artículos que contiene revela la destacada labor de los médicos y los investigadores del INP.

Es oportuno hacer la apología, el valor de la palabra escrita. Transcribo unas líneas del prólogo de un libro publicado por Méndez Editores sobre autores de la Medicina Mexicana del Siglo XX:

“... No basta que haya enseñado de viva voz dentro de las salas de hospital a la cabecera del 
lecho de los enfermos: no es suficiente que se impartan cursos y se realicen eventos científicos con participación de numerosos profesores y asistencia de cientos de estudiosos. Si todos los conocimientos vertidos en esas modalidades de enseñanza-aprendizaje quedan en la fase de discurso hablado, se perderá lo imperecedero de la palabra escrita; sin ella no se enterarían las generaciones jóvenes de los hechos de la historia, de los progresos, de las hipótesis y sus análisis".

El ser humano siempre ha tendido a dejar constancia de sus vivencias, desde que las plasmó haciendo grabados en las cavernas, hace miles de años, y gradualmente hasta la creación de los libros a raíz del desarrollo de la imprenta.

Infortunadamente, desde hace varios años ha declinado el interés por la lectura de libros. Esto es evidente respecto a los libros de medicina. Sé de dos empresas, una editorial de libros de medicina y otra dedicada a la venta de libros de distintas especialidades, que dejaron de publicar y de vender respectivamente sus libros. Ya no los compra la gente. ¿Cuáles son las causas?

Históricamente, la primera causa fue la proliferación de las ediciones "pirata". Era explicable que un estudiante de medicina con escasos recursos económicos optara por pagar la $5^{\mathrm{a}} \mathrm{o}$ la $6^{\mathrm{a}}$ parte del precio de un libro original, por una copia "pirata"; pero al menos, se supone que los alumnos todavía leían libros de texto.

La segunda causa, cuyo impacto es difícil cuantificar, es la que propició en su tiempo un director de la Escuela de Medicina, quien en sus listas de lecturas recomendadas daba los nombres de obras de medicina extranjeras, a pesar de que existían -y existen- libros de medicina mexicanos de buena calidad.

La tercera causa, seguramente la de mayor impacto, es el uso de la computadora. El estudiante recurre a ella y obtiene una enormidad

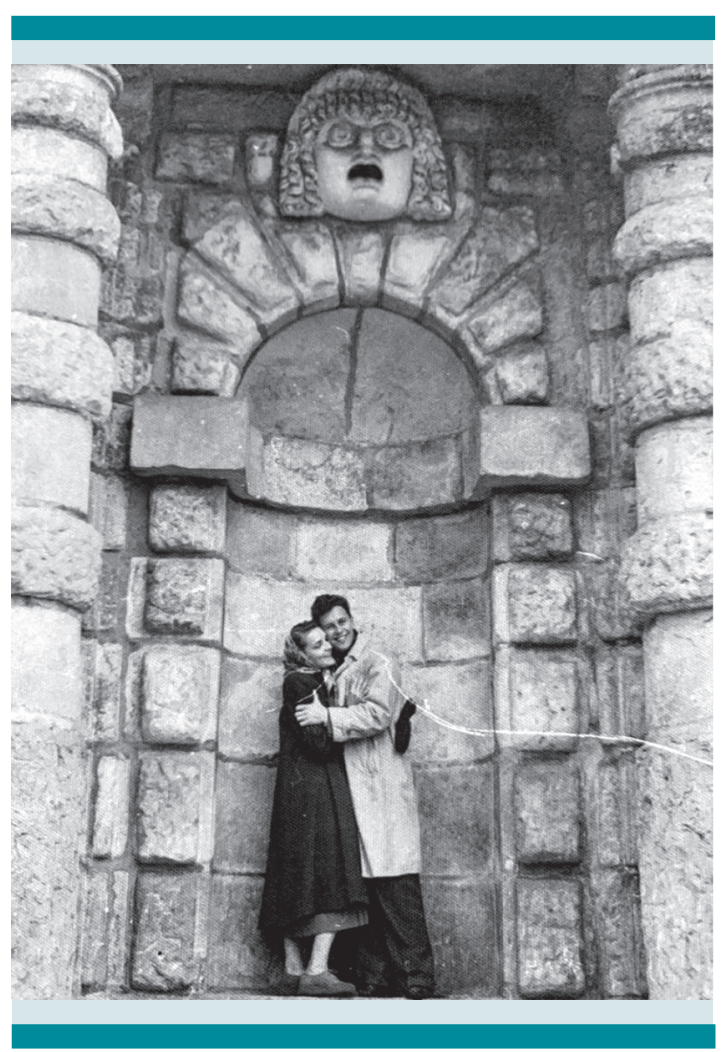

de conocimientos sin tener que consultar libros y por supuesto sin comprarlos. Hasta algunos profesores de la Facultad de Medicina recomiendan a sus alumnos consultar la computadora con preferencia a los libros. ¿Qué podemos decir quienes nos educamos leyendo libros? ¿Desaparecerán los libros de medicina? No lo sabemos.

Pienso que lo que proporciona la computadora es información, no educación. En este momento, como en muchos aspectos de nuestra vida, rige la ley del menor esfuerzo, de la línea con la menor resistencia; la propensión a obtener más sin tener que luchar por ello.

Ahora bien, un libro que contiene la experiencia del o de los autores, basada en sus años de ejercicio profesional tiene una vigencia limitada. Se apoya en los últimos conocimientos de la medicina; pero esa vigencia se pierde en un tiempo 
que varía de acuerdo con el lapso que tardan en aparecer nuevos hallazgos, nuevos medicamentos, nuevas técnicas. Aún así, perdurarán muchos descubrimientos, muchas verdades; pero el progreso es inevitable y significa cambios y avances continuos.

Surge entonces la justificación de una revista médica, que es a la vez libro periódico. Es libro

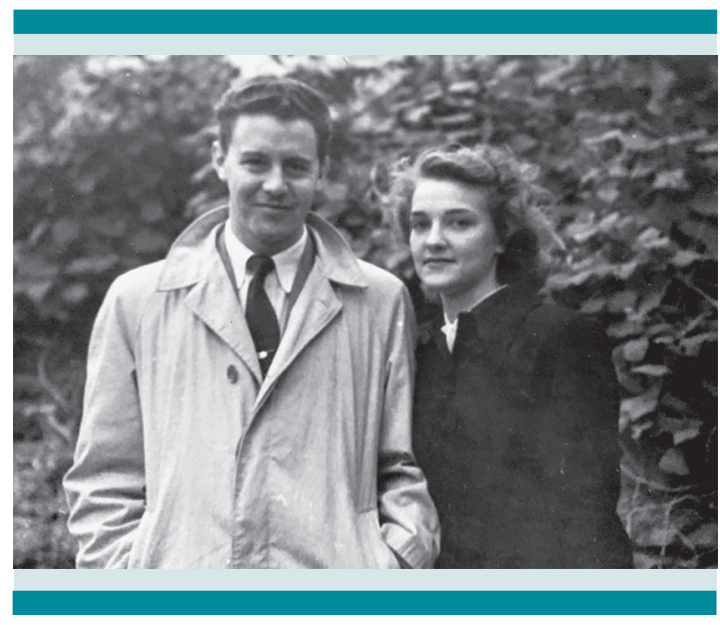

porque conserva la historia del quehacer médico y al mismo tiempo es periódico porque mantiene actualidad y dinamismo. Siempre está al día para difundir el pensamiento de sus autores. Para nuestra revista, esa difusión queda de manifiesto en las estadísticas que obtenemos de lectores o "visitas" cada mes, que son miles de numerosos países que consultan Acta Pediátrica de México.

Acta Pediátrica de México se puede consultar en varias páginas de internet y se encuentra en el Directorio de Literatura Latinoamericana de Ciencias de la Salud (LILACS); en el grupo II de la clasificación de Revistas Médicas de los Institutos Nacionales de Salud, en Artemisa, CENIDS y EBSCO (mediclatina).

En resumen, el hecho sobresaliente es el número elevado de lectores que la consultan para satisfacción y permanente estímulo de nuestros autores.

En la Unidad de Publicaciones continuamente pedimos artículos a nuestro médicos e investigadores, afortunadamente los recibimos. Es satisfactorio que cada vez más nos los envían de diversos estados del país y de otras instituciones.

Existe una enorme cantidad de material en el Archivo Clínico del INP, así como en los archivos propios de cada departamento. Todos son una fuente riquísima de información que pueden y deben explotar los médicos y los investigadores. Sin embargo, con cierta decepción, vemos que hay médicos de algunos servicios, cuya calidad y desempeño son innegables, que no han colaborado en la revista compartiendo sus conocimientos con una sola publicación en todos los años de vida del INP. Estos colegas no podrían decir que en su formación de médicos no les fue necesario leer libros ni consultar revistas.

Viene a la mente una corta sentencia en inglés dirigida a los investigadores, pero que puede aplicarse a los médicos de un instituto. Dice la sentencia: "Publish or Perish" o sea "Publica o muérete". No exageremos. Nadie tiene que morir por no publicar. Lo que se desea es que, congruente con la misión de un instituto -que no es un simple dispensario- los médicos no se limiten a su quehacer médico, sino que respondan a un reto: el compromiso moral de proyectar su labor educativa a través de contribuciones escritas.

Tomen en cuenta quienes no han tenido interés en escribir trabajos para la revista, que los miles de pediatras formados en el INP o en otros hospitales, al terminar sus estudios y regresar a su lugar de origen, desean estar al día en los avances de la medicina; que, para hacerlo, no sólo acuden una vez al año a los cursos de actualización de sus hospitales, sino que se informan constantemente en revistas como la nuestra. 
Para quienes sí publican su experiencia y prefieren hacerlo en inglés, hay que recordarles que en Latinoamérica hay millones de hispanoparlantes, miles de pediatras y de lectores de revistas médicas; que el español es una de las lenguas más habladas en el mundo.

A pesar de que Acta Pediátrica de México es la más leída entre las revistas pediátricas y consultada en muchos países, algunos de nuestros investigadores, quienes no ejercen la medicina en consultorios como lo hacen los clínicos y los cirujanos, con frecuencia deciden publicar sus estudios en una revista con una clasificación más alta, o en una revista extranjera, a fin de obtener mayor puntuación, lo que puede significarles recibir estímulos económicos.

Creo que hay motivos para aspirar a una mejor clasificación de nuestra revista, por sus cualidades y por sus 33 años de labor ininterrumpida creo justificado darle mucho valor a la producción de trabajos sobre medicina clínica y cirugía, y no situarla en una categoría inferior a la que al parecer se da a la investigación de laboratorio.
Hay que tomar en cuenta la proyección que Acta Pediátrica de México tiene en el universo de pediatras, a quienes se dirige la gran mayoría de los artículos que salen del Instituto Nacional de Pediatría. También hay que considerar la enorme población que abarca la Pediatría, que vela por la salud de sus pacientes y que comprende la atención de todos y cada uno de los órganos, aparatos y sistemas del individuo.

No es atributo del editor de una revista médica solicitar oficialmente y en forma razonada, un ascenso en la clasificación. Es asunto que compete probablemente a la autoridad de la institución. Entre tanto, seguiremos empeñados, cada cual, en su ámbito de acción, laborando en la mejor forma posible para proyectar y difundir el quehacer de nuestros médicos y nuestros investigadores, manteniéndonos al día, tratando de superarnos constantemente.

Descanse en paz Maestro Espino Vela. 